\title{
Traditional Acupuncture and Laser Acupuncture in Chronic Nonspecific Neck Pain: Study Protocol for a Randomized Controlled Trial
}

RAFAELA PERON ( $\square$ rafaelaperoncardoso@gmail.com )

Federal University of Sao Carlos: Universidade Federal de Sao Carlos https://orcid.org/0000-00018941-2885

\section{Érika Patrícia Rampazo}

Federal University of Sao Carlos: Universidade Federal de Sao Carlos

Richard Eloin Liebano

Federal University of Sao Carlos: Universidade Federal de Sao Carlos

\section{Research Article}

Keywords: Neck pain, Traditional Acupuncture, Laser Acupuncture, Study protocol, Randomized controlled trial

Posted Date: October 27th, 2021

DOl: https://doi.org/10.21203/rs.3.rs-853159/v1

License: (1) (i) This work is licensed under a Creative Commons Attribution 4.0 International License. Read Full License 


\section{Abstract}

Background: Nonspecific neck pain is a multifactorial and very common condition in adult individuals. No reports were found in the literature comparing the effectiveness of Traditional Acupuncture (TA) and Laser Acupuncture (LA) in cases of chronic nonspecific neck pain. Therefore, the aim of the present study is to investigate the effectiveness of TA and LA therapies in individuals with chronic nonspecific neck pain.

Methods/design: This study will be a controlled and randomized clinical trial. A total of 60 individuals will be randomized into two groups (30 each). The TA group will receive the acupuncture treatment using needles, while the LA group will receive the laser acupuncture treatment. The acupuncture points (Tianzhu, Fengchi, Jianjing, and Jianzhongshu) will be stimulated bilaterally with needles or laser. The primary outcome will be pain intensity, determined using the numerical rating scale. The secondary outcomes will be pressure pain threshold, temporal summation of pain, conditioned pain modulation, use of analgesic drugs after treatment, and the global perceived effect scale. The assessments will be performed immediately before and after treatment. At the follow-up, 1 months after the end of the treatments, evaluation will be made of the pain intensity and the global perceived effect. Statistical analysis of the data obtained will consider a significance level of $p<0.05$.

Discussion: This study will provide evidence concerning the effects of LA treatment, in comparison to TA, leading to benefits for individuals suffering from chronic nonspecific neck pain.

Trial registration: RBR-7vbw5gd (Brazilian Registry of Clinical Trials - ReBEC)

\section{Introduction}

\section{Background and rationale $\{6 a\}$}

Neck pain is a very common condition that causes considerable disability and economic losses $(1,2)$. Between the years 1990 and 2017, high indices of neck pain were reported in 195 countries, therefore it can be considered a serious global public health problem, ranked fourth among the causes of disability, with an annual prevalence rate higher than $30 \%(2,3)$. Neck pain is more common in women and usually increases with age, reaching peaks in the age groups 45-49 and 50-54 years for men and women, respectively, with a decrease for older persons (2). Several risk factors are associated with neck pain, including obesity and a sedentary lifestyle. Risk factors usually result in restrictions and disabilities that can have major impacts on the physical, social, and psychological well-being of the individual $(2,4,5)$. Neck pain can be classified according to its etiology. This kind of pain is considered specific when there are anatomical changes resulting from dysfunctions in neck structures, such as the deterioration of nerve roots, capsule damage, nerve root compression, or fractures (3). Nonspecific neck pain is multifactorial in nature and is not associated with a specific disease or anatomical modification $(6,7)$. Neck pain is classified according to the duration of symptoms, being acute (up to one month), subacute (one to three months), and chronic (at least three months) (8-10). Chronic neck pain is defined as any continuous pain 
in the cervical spine region between the base of the head and the upper shoulder, lasting at least 12 weeks and usually accompanied by reduced cervical movement (11).

Various strategies can be used for the treatment of neck pain, including traditional acupuncture (TA), which originated over 3,000 years ago and constitutes part of Traditional Chinese Medicine (12-15). The technique, performed with needles, produces good results and is effective in reducing pain and disability (16-23). TA provides clinical benefits in individuals with neck pain, being a viable option for use by health services (24). Furthermore, TA is considered a safe treatment, with serious side effects being rare, compared to conventional techniques used for the treatment of pain $(25,26)$.

There are now alternative acupuncture techniques available that do not use needles, such as laser acupuncture (LA), which combines use of the traditional Chinese acupuncture points with stimulation by laser light energy $(15,27,28)$. The lasers most indicated for LA are red or infrared, due to their deeper penetration into human tissue, with power outputs ranging from 5 to $500 \mathrm{~mW}(28-30)$. Laser acupuncture acts to enhance enzymatic defense systems, increasing plasma levels of superoxide dismutase (SOD), glutathione reductase (GRd), reduced glutathione (GSH), and catalase. LA assists in maintaining the integrity of the redox cycle and can promote reductions of malondialdehyde (MDA, a plasma marker of oxidative stress), the inflammation markers C-reactive protein (CRP) and interleukin-6 (IL-6), and glutathione peroxidase (GPx). Such alternative acupuncture technique can reduce oxidative stress damage, inflammation, joint edema, and pain in patients with rheumatoid arthritis (31).

An obvious difference between acupuncture using needles and LA is that the laser enables stimulation of acupuncture points without damaging the skin tissue (30). The laser induces a photochemical reaction in the cells, in a process known as photobiomodulation, at acupuncture points selected using the same rules as traditional Chinese acupuncture $(32,33)$. Using an optical power of around $40 \mathrm{~mW}$, the effect of LA is similar to that obtained with metal needles (28), being an attractive option for the treatment of chronic pain (34).

Acupuncture using needles is a widely accepted technique that provides good results and can be effective in reducing chronic neck pain $(16,17,19,20,35-45)$. Meanwhile, the clinical use of LA is increasing, since this technique offers the advantages of being totally painless, non-invasive, and safe $(30,33,43)$, which is appropriate for people who have phobia of needles. There are few reports in the scientific literature using AL for cervical pain condition $(39,46,47)$. However, until the present moment, no studies were found that compared traditional acupuncture with LA in patients with chronic nonspecific neck pain. There is a need for thorough research concerning $L A$, with robust methodologies, large sample sizes, and standardized and reproducible parameters, in order to increase understanding of this therapy and establish possible future clinical applications $(30,48,49)$.

\section{Objectives $\{7\}$}

The aim of this study is to compare the effects of Traditional Acupuncture and Laser Acupuncture therapies in individuals with chronic nonspecific neck pain. 


\section{Trial design $\{8\}$}

This study will be a randomized clinical trial (RCT) conducted according to the established standards for reporting clinical trials: CONSORT (Consolidated Standards of Reporting Trials) and STRICTA (Standards for Reporting Interventions in Clinical Trials of Acupuncture). The protocol was developed following the recommendations of SPIRIT (Standard Protocol Items: Recommendations for Interventional Trials). The RCT will have a blinded evaluator and the acupuncture styles adopted will be Traditional Acupuncture and Laser Acupuncture.

\section{Methods}

\section{Participants, interventions and outcomes}

\section{Study setting \{9\}}

The study will be undertaken at the Physiotherapy Department of the Federal University of São Carlos (UFSCar), in the city of São Carlos, São Paulo State, Brazil.

\section{Eligibility criteria $\{10\}$}

After screening using an online form, the potential participants will be registered and checks will be performed considering the inclusion and exclusion criteria.

\section{Inclusion criteria}

- Individuals with complaints of nonspecific neck pain, reported during the screening;

- Neck pain for at least 3 months;

- Numerical rating scale (NRS) score $\geq 3$ for pain intensity;

- Age between 18 and 60 years;

- Men and women;

- Completed primary education;

\section{Exclusion criteria}

- Fracture or history of spinal injury;

- Spondylolisthesis;

- Stenosis of the spinal canal;

- Ankylosing spondylitis;

- Signs of radiculopathy;

- Cancer;

- Acute infections; 
- Hemorrhagic disease;

- Rheumatological disease;

- Systemic inflammatory disease;

- Rheumatoid arthritis;

- Alteration in skin sensitivity;

- Hearing difficulty (without correction);

- Difficulty in answering questionnaires;

- Pregnancy, puerperium, and breastfeeding;

- Individuals who have started or been submitted to any type of intervention or treatment in the three months prior to the date of screening.

- Individuals who do not accept to remain in the ventral decubitus position, upper limbs along the body and cervical in neutral position, during the time of interventions.

- Individuals who do not having ingested any sedative, anti-inflammatory and analgesic medications (narcotics and non-narcotics), and alcoholic substance in the last 48 hours before the intervention.

\section{Who will take informed consent? $\{26 \mathrm{a}\}$}

Data collection will only start after the participants have signed an informed consent form, which will be explained by the evaluator.

\section{Additional consent provisions for collection and use of participant data and biological specimens \{26b\}}

Not applicable for this study.

\section{Interventions}

\section{Explanation for the choice of comparators $\{6 \mathrm{~b}\}$}

No studies were found in the literature that effectively compared Traditional Acupuncture with Laser Acupuncture for treatment of chronic nonspecific neck pain. Therefore, the proposed research will focus on evaluating and comparing changes in pain symptoms and neck functional capacity, after application of TA and LA in cases of chronic nonspecific neck pain.

\section{Intervention description $\{11 \mathrm{a}\}$}

The interventions will follow a standardized therapy protocol. Four acupuncture points (Tianzhu, Fengchi, Jianjing, and Jianzhongshu) will be stimulated bilaterally with needles or laser. The participant will be positioned in a ventral decubitus position, upper limbs along the body and cervical in neutral position, asked to rest for 5 minutes, prior to the intervention. At the locations where the needles or laser will be applied, the skin will be hygienized with gauze and $70 \%$ alcohol solution. A dermatograph pencil (COLOURED ${ }^{\circledR}$ ) will be used to draw circles with diameters of $2 \mathrm{~cm}$ around the application points. The 
same researcher, an acupuncturist with specialization in the field and 9 years of clinical experience, will perform all the interventions. The individuals will be asked not to use any other treatment for neck pain during the study period. All the data collected about the participants will be confidential.

\section{Traditional Acupuncture (TA)}

For the TA group, the interventions will be performed using disposable Goldlife ${ }^{\circledR}$ needles $(0.25 \mathrm{~mm}$ diameter, $40 \mathrm{~mm}$ length; Brazilian product registration number MS80553910002) supplied in packages containing 10 surgical steel needles and a needle guide tube. Using the guide tube, the needles will be appropriately positioned in the centers of the areas demarcated on the skin and manually inserted into the muscle to a depth of $20 \mathrm{~mm}$. The acupuncture points (Tianzhu, Fengchi, Jianjing, and Jianzhongshu) will be stimulated bilaterally. The manual needle stimulation technique known as sparrow pecking will be used $(35,50,51)$. After the stimuli, the needles will be left in position for 20 minutes $(43,47,52)$, followed by removal using a stainless-steel collecting cup and disposal in an appropriate container for sharps (Descarpack ${ }^{\circledR}$ ), in each session new needles will be used.

\section{Laser Acupuncture (LA)}

For the LA group, the interventions will be performed using an Antares ${ }^{\circledR}$ laser (IBRAMED, Amparo, São Paulo, Brazil). The laser probe will be appropriately positioned at the locations demarcated on the skin, the acupuncture points: Tianzhu; Fengchi; Jianjing; Jianzhongshu. The dose (Table 1) being based on previous work using LA for neck pain (47), following the criteria suggested by Baxter (49).

\section{Criteria for discontinuing or modifying allocated interventions $\{11 \mathrm{~b}\}$}

There will be no changes in the allocations and no migration of individuals between groups will be allowed. If individuals discontinue treatment, recent data will be computed for the analyses according to the intention-to-treat principle, and the motive for the desistence will be recorded.

\section{Strategies to improve adherence to interventions $\{11 \mathrm{c}\}$}

To minimize data loss, all the participants in the trial will receive a card with the date and time of session. The therapist responsible for the session will send a WhatsApp message from a cellphone account used only for this research, confirming the session, one day in advance. For the evaluations and reevaluations, the blinded evaluator will contact the participants by WhatsApp, confirming the initial and final evaluations, as well as the follow-up 1 months after the end of the intervention, which will be performed by telephone. In cases of abandonment or impossibility of continuing the study, the data will be analyzed according to an intention-to-treat protocol.

\section{Relevant concomitant care permitted or prohibited during the trial $\{11 \mathrm{~d}\}$}

Concomitant treatments such as continuous use medications for hypertension and diabetes will be permitted during the trial. The participants will not be permitted to start any new type of intervention or 
activity during the trial period.

\section{Provisions for post-trial care $\{30\}$}

A follow-up will be performed by telephone, 1 month after the end of the intervention.

\section{Outcomes $\{12\}$}

The primary outcome will be the pain intensity before-after data. It will be evaluated using the numerical rating scale (NRS). Secondary outcomes will be as follows: pressure pain threshold (PPT); temporal summation of pain (TS); conditioned pain modulation (CPM); use of analgesic drugs after treatments; and Global Perceived Effect Scale (GPE). These outcomes will be evaluated before and after the treatments. Evaluation using the NRS and the global perceived effect scale will be performed at the end of the 1-month follow-up period.

\section{Numeric rating scale (NRS)}

The NRS employs a simple and easy to use scale, where the individual is asked to indicate the intensity of the current pain, using a scale ranging from 0 ("no pain") to 10 ("worst pain imaginable") (53). The participants will be instructed to rate the pain experienced at rest and during movements (flexion, extension, lateral inclination to the left and right, and rotation to the left and right). All before-after data will be individually recorded and compared for their respectively positions.

\section{Pressure pain threshold (PPT)}

Measurements will be made using a pressure algometer (Somedic ${ }^{\circledR}$, Hörby, Sweden), with the tip of the instrument $\left(1 \mathrm{~cm}^{2}\right)$ positioned on cervical zygapophysial joints $\mathrm{C5} / 6(54)$, between $\mathrm{C} 7$ and the acromion, bilaterally at the midpoint of the upper trapezius muscle (55), and on the middle third of the right tibialis anterior muscle. All these points will be previously marked with a dermatograph pencil. The algometer probe will be positioned perpendicular to the skin and pressure will be applied at a rate of $40 \mathrm{kPa} / \mathrm{s}$. Three consecutive PPT measurements will be made, with an interval of $30 \mathrm{~s}$ between them, and the average value will be recorded. The participants will be instructed to close their eyes and to press the algometer sensor when the pressure sensation becomes painful, with the corresponding value being recorded. A test will be performed to familiarize the individuals with the algometer, explaining the procedure, and a demonstration will be made using the thenar region of the hand. During the actual measurements, the individuals will not be allowed to see the algometer. The intra-examiner reliability of the PPT measurements will be evaluated using measurements at the same points for 10 healthy and asymptomatic individuals, performed by a single examiner at intervals of 48 hours. The reliability will then be estimated by calculating intraclass correlation coefficients (ICC) (54-57).

\section{Temporal summation of pain (TS)}


Measurements will be made using the pressure algometer (Somedic ${ }^{\circledR}$, Hörby, Sweden), applying a total of 10 stimuli, at a rate of $40 \mathrm{kPa} / \mathrm{s}$, using the average value from the previous algometry test performed on the most painful upper trapezius muscle (or the dominant side if there is no evident difference). Each stimulus will be maintained for $1 \mathrm{~s}$, using a timer to ensure the correct intervals $(1 \mathrm{~s})$. The individuals will be asked about their pain at the $1^{\text {st }}, 5^{\text {th }}$, and $10^{\text {th }}$ seconds of stimulus, using the NRS. In order to avoid any interference, the test will begin 5 minutes after the PPT $(57,58)$.

\section{Conditioned pain modulation (CPM)}

Cold nociceptive stimulus will be employed. Individuals will receive instructions before the test. Conditioned stimulus will be provided by the hand on the side ipsilateral to the region of the greatest neck pain, which will be immersed for 1 minute in a water bath kept at $22^{\circ} \mathrm{C}$, in order to standardize the hand temperature. After that, the same hand will be submerged (up to the wrist) for 1 minute in ice water (kept at $4{ }^{\circ} \mathrm{C}$ ). After 30 seconds, the individual will be asked to report (using the NRS) the intensity of the pain in the hand, caused by the ice water. After immersion for 1 minute, the patient will be asked to remove the hand from the ice water. The PPT will be performed (between C7 and the acromion, at the mid-point of the upper trapezius muscle), using the side contralateral to the immersed hand. The PPT evaluation will be performed before and immediately after the cold stimulus. The mean PPT value before the stimulus will be subtracted from the mean value after the stimulus, with a lower value indicating that the endogenous system is less efficient in inhibiting pain $(57,59,60)$.

\section{Use of analgesic drugs}

It will be important to record the use of analgesic drugs, in order to determine whether their use decreased after treatment. The participants will be asked to report, all the medications used. This information will be collected and recorded. In tables, the analgesic medications will be converted into opioids, considering the equianalgesic dose of morphine (61), and non-opioids, considering the equianalgesic dose of acetaminophen (paracetamol) (62).

\section{Global perceived effect scale (GPE)}

The global perceived effect scale is used to assess the perception of the individual regarding any improvement, comparing the initial symptoms with the current condition. This scale, which has been translated into Portuguese and validated, provides good reliability when applied to musculoskeletal disorders. It uses 11 points, ranging from -5 (much worse), through 0 (no change), to 5 (completely recovered). After treatment, the participants will be asked: "Compared to when this problem first started, how would you describe the condition of your neck today?" A higher score indicates an improved condition $(63,64)$.

\section{Participant timeline $\{13\}$}

\section{Sample size $\{14\}$}


The calculation of the sample size considered a difference of 1.5 points between groups, which would be a clinically relevant value for the outcome of neck pain intensity, as measured using the numeric rating scale (NRS) (65), with an estimated standard deviation of 1.6 points (37). Statistical power of $90 \%$ and a possible sample loss of $20 \%$ were considered. Consequently, a total of 60 individuals will be needed, with 30 in each group. This calculation was performed using Minitab v.17 software (State College, PA, USA).

\section{Recruitment \{15\}}

Recruitment of participants in the research will be achieved by means of announcements using local media, internet, radio, and posters distributed throughout the city of São Carlos.

\section{Assignment of interventions: allocation}

\section{Sequence generation $\{16 a\}$}

The individuals included in the study will be randomized using block exchange methods available at the website http://randomization.com, with allocation to the TA group $(n=30)$ or the LA group $(n=30)$.

\section{Concealment mechanism \{16b\}}

Concealment of the allocation will be achieved using sequential numbering, in sealed opaque envelopes, which will only be opened before the first session, by the researcher responsible for application of the treatments.

\section{Implementation \{16c\}}

Randomization will be performed by a researcher responsible only for the randomization, with no other role in this study.

\section{Assignment of interventions: Blinding}

\section{Who will be blinded $\{17 \mathrm{a}\}$}

The evaluator will be blinded to the randomization and intervention processes, be responsible only for recruitment and evaluations, and receive no information regarding the allocation of the individuals to the groups. The participants and the researcher responsible for the treatment will not be blinded, due to the nature of the interventions.

\section{Procedure for unblinding if needed $\{17 b\}$}

The evaluator will not be allowed to unblock the blinding. However, the acupuncturist researcher responsible for applying the treatments will not be blinded.

\section{Data collection and management}




\section{Plans for assessment and collection of outcomes $\{18 \mathrm{a}\}$}

The interventions will follow an application protocol. The evaluator will be trained in application of the questionnaires and tests, while intra-examiner reliability will be estimated by calculation of ICC.

\section{Plans to promote participant retention and complete follow-up $\{18 \mathrm{~b}\}$}

The participants will be provided with guidance when they sign the informed consent form and commit to attending on the scheduled treatment dates. They will receive a card with the dates of the treatment sessions, a contact telephone number, and a WhatsApp contact number used only for this research. This procedure will ensure that participants receive the necessary attention and will assist in fully accompanying them during the research. If individuals abandon the trial, the reasons will be recorded, the most recent data will be compiled, and analysis will be performed using the intention-to-treat principle.

\section{Data management $\{19\}$}

All the data will be collected weekly, saved as PDF files, and stored on the UFSCar institutional Google Drive. The person responsible for data management will be the principal investigator, namely the acupuncturist researcher who applies the treatments.

\section{Confidentiality $\{27\}$}

The data will be stored on the institutional Google Drive of Federal University of São Carlos (UFSCar). Only researchers from the UFSCar, who participate in this research, will have authorization to access these stored documents. The metadata will be stored for the long term in the UFSCar research data repository.

\section{Plans for collection, laboratory evaluation and storage of biological specimens for genetic or molecular analysis in this trial/future use $\{33\}$}

Not applicable to this research.

\section{Statistical methods}

\section{Statistical methods for primary and secondary outcomes $\{20 \mathrm{a}\}$}

The statistical inferences for the primary and secondary outcomes will be made by an assessor blinded to the randomization and assessment processes. The intention-to-treat principle will be adopted in the analyses. The normality of the data will be evaluated using the Kolmogorov-Smirnov test. Specific analyses will be used, depending on the distribution (normality and homogeneity) of the data. Parametric and nonparametric analyses will be used for normally and non-normally distributed data, respectively. These analyses will be performed using SPSS v.17 statistical software (SPSS, Inc., IL, USA), considering a statistical power of $90 \%$ for all the tests, with a significance level of $p<0.05$. 
Interim analyses $\{21 \mathrm{~b}\}$

No provisional analyses will be performed. In cases of discontinuity, the intention-to-treat principle will be adopted.

Methods for additional analyses (e.g. subgroup analyses) \{20b\}

No subgroups will be analyzed.

Methods in analysis to handle protocol non-adherence and any statistical methods to handle missing data $\{20 \mathrm{c}\}$

In the case of discontinuity, missing data will be handled by adopting the intention-to-treat principle, in order to make statistical inferences.

Plans to give access to the full protocol, participant level-data and statistical code $\{31 \mathrm{c}\}$

Not applicable to this research.

\section{Oversight and monitoring}

Composition of the coordinating center and trial steering committee $\{5 \mathrm{~d}\}$

Not applicable to this research.

Composition of the data monitoring committee, its role and reporting structure $\{21 a\}$

Data monitoring committees are usually established for large studies. The present study is not multicenter and will be performed at UFSCar. The researcher will be responsible for organizing and monitoring the data obtained in this research.

\section{Adverse event reporting and harms $\{22\}$}

In the case of any harm or complication related to the treatments, this will be reported to the UFSCar Ethics Committee for Human Research. The same applies to possible ethical issues that may arise during the research. The guideline recommendations will be strictly followed.

Frequency and plans for auditing trial conduct $\{23\}$

Not applicable to this research.

Plans for communicating important protocol amendments to relevant parties (e.g. trial participants, ethical committees) $\{25\}$

This study it was registered with RBR-7vbw5gd (Brazilian Registry of Clinical Trials - ReBEC) and there will be no substantial alterations to the protocol, such as changes in the eligibility criteria and analyses. If 
there is any modification, it will be reported in writing, in a document sent to the trial registration office.

\section{Dissemination plans $\{31$ a $\}$}

After publication of the results in a scientific journal, all data will be available over the long term and kept in the UFSCar research data repository.

\section{Discussion}

The purpose of this study is to compare the TA and LA techniques, assessing which is most effective in reducing chronic nonspecific neck pain. Since the 1970s, the World Health Organization (WHO) has encouraged the use of TA (66), considered to be a low-cost therapy (24) that can provide clinical benefits relevant to neck pain $(19,36)$. In contrast, there have been few studies of the use of LA for conditions involving neck pain (47). LA is an efficient analgesic and anti-inflammatory therapy that is totally painless, non-invasive, safe, and without adverse effects or complications. This technique may be used in locations where the insertion of needles is complicated or risky $(30,33,43)$, being a useful option for individuals with needle phobia. Until the present moment, there are no studies in the literature that have effectively compared TA and LA in cases of chronic nonspecific neck pain.

This research will be based on evaluating and comparing changes in pain symptoms and the functional capacity of the neck, after application of the treatments. The results obtained for the two groups will be quantified and compared. Based on the findings, it will be possible to establish the therapeutic potential of each treatment, considering the outcomes of pain and cervical disability, making possible to identify whether there is a difference between the techniques in the treatment of chronic nonspecific neck pain.

\section{Trial status}

The protocol registration was approved on 6th June 2021. The initial recruitment date will be 10th August 2021 and the approximate date of completion of the recruitment of participants will be up to the second half of 2022.

\section{Abbreviations}

Global Perceived Effect Scale (GPE)

Conditioned Pain Modulation (CPM)

Temporal summation of pain (TS)

Pressure Pain Threshold (PPT)

Numerical Rating Scale (NRS)

Traditional Acupuncture (TA) 
Laser Acupuncture (LA)

Federal University of São Carlos (UFSCar)

Brazilian Registry of Clinical Trials (ReBEC)

\section{Declarations}

\section{Acknowledgements}

Coordenação de Aperfeiçoamento de Pessoal de Nível Superior - Brasil (CAPES - Finance code 001).

\section{Authors' contributions \{31b\}}

Peron, R.: Conceptualization, Methodology, Writing original draft.

Rampazo, É. P.: Methodology, Review.

Liebano, R. E.: Conceptualization, Methodology, Review, Supervision.

All authors have given final approval of the version to be published.

\section{Funding $\{4\}$}

This work will be financed in part by the Coordenação de Aperfeiçoamento de Pessoal de Nível Superior Brazil (CAPES - Finance code 001), PhD Scholarships. Therefore, this works does not receive any research funding for the development of the study.

\section{Availability of data and materials $\{29\}$}

Not applicable.

\section{Ethics approval and consent to participate $\{24\}$}

The authors declare that they have obtained the approval of the Research Ethics Committee of the Federal University of São Carlos (UFSCar), under protocol number CAAE 39912520.1.0000.5504. In order to participate in this research, the volunteers must sign free and informed consent forms.

\section{Consent for publication $\{32\}$}

Not applicable.

\section{Competing interests $\{28\}$}

There are no conflicts of interest.

\section{Authors' information (optional)}




\section{References}

1. Henschke N, Kamper SJ, Maher CG. The epidemiology and economic consequences of pain. Mayo Clin Proc [Internet]. 2015;90(1):139-47. Available from: http://dx.doi.org/10.1016/j.mayocp.2014.09.010.

2. Safiri S, Kolahi AA, Hoy D, Buchbinder R, Mansournia MA, Bettampadi D, et al. Global, regional, and national burden of neck pain in the general population, 1990-2017: Systematic analysis of the Global Burden of Disease Study 2017. BMJ. 2020;368.

3. Cohen SP. Epidemiology, diagnosis, and treatment of neck pain. Mayo Clin Proc [Internet]. 2015;90(2):284-99. Available from: http://dx.doi.org/10.1016/j.mayocp.2014.09.008.

4. Genebra CVDS, Maciel NM, Bento TPF, Simeão SFAP, Vitta A, De. Prevalence and factors associated with neck pain: a population-based study. Brazilian J Phys Ther [Internet]. 2017;21(4):274-80. Available from: http://dx.doi.org/10.1016/j.bjpt.2017.05.005.

5. Sun M, Geng G, Chen J, Ma X, Yan M, Liu X, et al. Acupuncture for chronic neck pain with sensitive points: Study protocol for a multicentre randomised controlled trial. BMJ Open. 2019;9(7):1-8.

6. Misailidou V, Malliou P, Beneka A, Karagiannidis A, Godolias G. Assessment of patients with neck pain: a review of definitions, selection criteria, and measurement tools. J Chiropr Med [Internet]. 2010;9(2):49-59. Available from: http://dx.doi.org/10.1016/j.jcm.2010.03.002.

7. Silva ACDO, Biasotto-Gonzalez DA, Oliveira FHM, Andrade AO, Gomes CAFDP, Lanza FDC, et al. Effect of Osteopathic Visceral Manipulation on Pain, Cervical Range of Motion, and Upper Trapezius Muscle Activity in Patients with Chronic Nonspecific Neck Pain and Functional Dyspepsia: A Randomized, Double-Blind, Placebo-Controlled Pilot Study. Evidence-based Complement Altern Med. 2018;2018.

8. Kjaer P, Kongsted A, Hartvigsen J, Isenberg-Jørgensen A, Schiøttz-Christensen B, Søborg B, et al. National clinical guidelines for non-surgical treatment of patients with recent onset neck pain or cervical radiculopathy. Eur Spine J. 2017;26(9):2242-57.

9. Kroeling P, Gross A, Graham N, Burnie SJ, Szeto G, Goldsmith CH, et al. Electrotherapy for neck pain. Cochrane database Syst Rev. 2013;8.

10. Blanpied PR, Gross AR, Elliott JM, Devaney LL, Clewley D, Walton DM, et al. Clinical practice guidelines linked to the international classification of functioning, disability and health from the orthopaedic section of the American physical therapy association. J Orthop Sports Phys Ther. 2017;47(7):A1-83.

11. Martimbianco ALC, Porfírio GJM, Pacheco RL, Torloni MRRR. Transcutaneous electrical nerve stimulation (TENS) for chronic neck pain. Cochrane Database Syst Rev. 2019;(12).

12. Jiang X. Effects of magnetic needle acupuncture on blood pressure and plasma ET-1 level in the patient of hypertension. J Tradit Chinese Med. 2003;23(4):290-1.

13. Cabýoglu MT, Ergene N, Tan U. The mechanism of acupuncture and clinical applications. Int $J$ Neurosci. 2006;116(2):115-25. 
14. Kotzé A, Simpson KH. Stimulation-produced analgesia: acupuncture, TENS and related techniques. Anaesth Intensive Care Med. 2008;9(1):29-32.

15. Lim T-K, Ma Y, Berger F, Litscher G. Acupuncture and Neural Mechanism in the Management of Low Back Pain-An Update. Vol. 5, Medicines. 2018. 63 p.

16. Irnich D, Behrens N, Gleditsch JM, Stör W, Schreiber MA, Schöps P, et al. Immediate effects of dry needling and acupuncture at distant points in chronic neck pain: Results of a randomized, doubleblind, sham-controlled crossover trial. Pain. 2002;99(1-2):83-9.

17. Vas J, Perea-Milla E, Méndez C, Navarro CS, León Rubio JM, Brioso M, et al. Efficacy and safety of acupuncture for chronic uncomplicated neck pain: A randomised controlled study. Pain. 2006;126(13):245-55.

18. Salter GC, Roman M, Bland MJ, MacPherson H. Acupuncture for chronic neck pain: A pilot for a randomised controlled trial. BMC Musculoskelet Disord. 2006;7:1-9.

19. Fu LM, Li JT, Wu WS. Randomized controlled trials of acupuncture for neck pain: Systematic review and meta-analysis. J Altern Complement Med. 2009;15(2):133-45.

20. Yuan QL, Guo TM, Liu L, Sun F, Zhang YG. Traditional chinese medicine for neck pain and low back pain: A systematic review and meta-analysis. PLoS One [Internet]. 2015;10(2):1-37. Available from: http://dx.doi.org/10.1371/journal.pone.0117146.

21. Trinh K, Graham N, Irnich D, Id C, Forget M. Acupuncture for neck disorders (Review) Summary of findings for the main comparison. 2016;(5).

22. Ho LF, Lin ZX, Leung AWN, Chen L, Zhang H, Ng BFL, et al. Efficacy of abdominal acupuncture for neck pain: A randomized controlled trial. PLoS One. 2017;12(7):1-18.

23. Coan R, Wong GC. The acupuncture treatment of neck pain: A randomized controlled study. Am J Chin Med. 1982;9:326-32.

24. Willich SN, Reinhold T, Selim D, Jena S, Brinkhaus B, Witt CM. Cost-effectiveness of acupuncture treatment in patients with chronic neck pain. Pain. 2006;125(1-2):107-13.

25. Dung H. Acupuncture: An Anatomical Approach. London, UK; 2014. Chapter 9.

26. Patil S, Sen S, Bral M, Reddy S, Bradley KK, Cornett EM, et al. The Role of Acupuncture in Pain Management. Curr Pain Headache Rep. 2016;20(4):1-8.

27. Litscher G. High-tech laser acupuncture is Chinese medicine. Med Acupunct. 2008;20(4):245-54.

28. Litscher G, Opitz G. Technical parameters for laser acupuncture to elicit peripheral and central effects: State-of-the-art and short guidelines based on results from the Medical University of Graz, the German Academy of Acupuncture, and the scientific literature. Evidence-based Complement Altern Med. 2012;2012(February).

29. Round R, Litscher G, Bahr F. Auricular acupuncture with laser. Evidence-based Complement Altern Med. 2013;2013.

30. Chon TY, Mallory MJ, Yang J, Bublitz SE, Do A, Dorsher PT. Laser Acupuncture: A Concise Review. Med Acupunct. 2019;31(3):164-8. 
31. Attia AMM, Ibrahim FAA, Abd El-Latif NA, Aziz SW, Elwan AM, Abdel Aziz AAA, et al. Therapeutic antioxidant and anti-inflammatory effects of laser acupuncture on patients with rheumatoid arthritis. Lasers Surg Med. 2016;48(5):490-7.

32. Whittaker P. Laser acupuncture: Past, present, and future. Lasers Med Sci. 2004;19(2):69-80.

33. Wu S-Y, Kuo C-E, Hung Y-C, Hu W-L. Managing Pain with Laser Acupuncture. Pain Manag. 2016.

34. Zeng YJ, Lin YH, Wang YC, Chang JH, Wu JH, Hsu SF, et al. Laser acupuncture-induced analgesic effect and molecular alterations in an incision pain model: a comparison with electroacupunctureinduced effects. Lasers Med Sci. 2018;33(2):295-304.

35. Nabeta T, Kawakita K. Relief of chronic neck and shoulder pain by manual acupuncture to tender points - A sham-controlled randomized trial. Complement Ther Med. 2002;10(4):217-22.

36. Trinh K, Graham N, Irnich D, Cameron ID, Forget M. Acupuncture for neck disorders. Cochrane Database Syst Rev. 2016;2016(5):236-43.

37. Tsai CT, Hsieh LF, Kuan TS, Kao MJ, Chou LW, Hong CZ. Remote effects of dry needling on the irritability of the myofascial trigger point in the upper trapezius muscle. Am J Phys Med Rehabil. 2010;89(2):133-40.

38. Wong RHL, Lee TW, Sihoe ADL, Wan IYP, Ng CSH, Chan SKC, et al. Analgesic Effect of Electroacupuncture in Postthoracotomy Pain: A Prospective Randomized Trial. Ann Thorac Surg. 2006;81(6):2031-6.

39. Seidel UUC. A randomised controlled double-blind trial comparing dosed laser therapy on acupuncture points and acupuncture for chronic cervical syndrome [in German]. Dtsch Zeitschri(fur Akupunkt. 2002;45:258-69.

40. Cameron ID, Wang ESD. A randomized trial comparing acupuncture and simulated acupuncture for subacute and chronic whiplash. Spine (Phila Pa 1976). 2011;36:1659-65.

41. Chou LW, Hsieh YL, Kao MJ, Hong CZ. Remote Influences of Acupuncture on the Pain Intensity and the Amplitude Changes of Endplate Noise in the Myofascial Trigger Point of the Upper Trapezius Muscle. Arch Phys Med Rehabil [Internet]. 2009;90(6):905-12. Available from: http://dx.doi.org/10.1016/j.apmr.2008.12.020.

42. Coan RM, Wong GCP. The acupuncture treatment of neck pain: a randomized controlled study. Am J Chin Med. 1982;32(4):326.

43. Eslamian F, Hajialilo M, Mohammadi L. Comparison of Therapeutic Efficacy of Acupuncture and Low-Level Laser in the Treatment of Cervical Myofascial Pain Syndrome: A Single-Blind Randomized Controlled Clinical Trial. Phys Med Rehab Electrodiagnosis. 2019;1:14-24.

44. He D, Hostmark AT, Veiersted KBMJ. EFect of intensive acupuncture on pain-related social and psychological variables for women with chronic neck and shoulder pain - an RCT with six month and three year follow up. Acupunct Med. 2005;23:52-61.

45. Yuan QL, Guo TM, Liu L, Sun F, Zhang YG. Traditional chinese medicine for neck pain and low back pain: A systematic review and meta-analysis. PLoS One. 2015;10(2):1-37. 
46. Ilbuldu E, Cakmak A, Disci R, Aydin R. Comparison of laser, dry needling, and placebo laser treatments in myofascial pain syndrome. Photomed Laser Surg. 2004;22(4):306-11.

47. El-Kharbotly AM, El-Gendy AA, Mohammed MA, El-Masry MR, Daoud EM, Hassan N, et al. Effect of laser acupuncture versus traditional acupuncture in neck pain of cervical spondylosis. Mech LowLight Ther IX. 2014;8932:89320Z.

48. Liebert MA. Consensus agreement on the design and conduct of clinical studies with low-level laser therapy and light therapy for musculoskeletal pain and disorders. Photomed Laser Surg. 2006;24(6):761-2.

49. Baxter GD, Bleakley C, McDonough S. Clinical effectiveness of laser acupuncture: A systematic review. JAMS J Acupunct Meridian Stud [Internet]. 2008;1(2):65-82. Available from: http://dx.doi.org/10.1016/S2005-2901(09)60026-1.

50. Inoue M, Kitakoji H, Ishizaki N, Tawa M, Yano T, Katsumi Y, et al. Relief of low back pain immediately after acupuncture treatment - A randomised, placebo controlled trial. Acupunct Med. 2006;24(3):103-8.

51. Itoh K, Katsumi Y, Hirota S, Kitakoji H. Randomised trial of trigger point acupuncture compared with other acupuncture for treatment of chronic neck pain. Complement Ther Med. 2007;15(3):172-9.

52. Liang Z, Zhu X, Yang X, Fu WLA. Assessment of a traditional acupuncture therapy for chronic neck pain: a pilot randomised controlled study. Complement Ther Med. 2011;195:526-32.

53. Young IA, Cleland JA, Michener LA, Brown C. Reliability, construct validity, and responsiveness of the neck disability index, patient-specific functional scale, and numeric pain rating scale in patients with cervical radiculopathy. Am J Phys Med Rehabil. 2010;89(10):831-9.

54. Lluch E, Schomacher J, Gizzi L, Petzke F, Seegar D, Falla D. Immediate effects of active craniocervical flexion exercise versus passive mobilisation of the upper cervical spine on pain and performance on the cranio-cervical flexion test. Man Ther [Internet]. 2014;19(1):25-31. Available from: http://dx.doi.org/10.1016/j.math.2013.05.011.

55. Celenay ST, Kaya DO, Akbayrak T. Cervical and scapulothoracic stabilization exercises with and without connective tissue massage for chronic mechanical neck pain: A prospective, randomised controlled trial. Man Ther [Internet]. 2016;21(April 2018):144-50. Available from: http://dx.doi.org/10.1016/j.math.2015.07.003.

56. Almeida N, Paladini LH, Korelo RIG, Liebano RE, de Macedo ACB. Immediate Effects of the Combination of Interferential Therapy Parameters on Chronic Low Back Pain: A Randomized Controlled Trial. Pain Pract. 2020;20(6):615-25.

57. Rampazo ÉP, de Andrade ALM, da Silva VR, Back CGN, Liebano RE. Photobiomodulation therapy and transcutaneous electrical nerve stimulation on chronic neck pain patients. Medicine. 2020;99(8):e19191.

58. Corrêa JB, et al. Effects of the carrier frequency of interferential current on pain modulation and central hypersensitivity in people with chronic nonspecific low back pain: a randomized placebocontrolled trial. Eur J Pain. 2016;20:1653-66. 
59. Yarnitsky D, Bouhassira D, Drewes AM, Fillingim RB, Granot M, Hansson P, et al. Recommendations on practice of conditioned pain modulation (CPM) testing. Eur J Pain (United Kingdom). 2015;19(6):805-6.

60. Imai Y, Petersen KK, Mørch CD, Arendt Nielsen L. Comparing test-retest reliability and magnitude of conditioned pain modulation using different combinations of test and conditioning stimuli. Somatosens Mot Res [Internet]. 2016;33(3-4):169-77. Available from: http://dx.doi.org/10.1080/08990220.2016.1229178.

61. Knotkova H, Fine PG, Portenoy RK. Opioid Rotation: The Science and the Limitations of the Equianalgesic Dose Table. J Pain Symptom Manage [Internet]. 2009;38(3):426-39. Available from: http://dx.doi.org/10.1016/j.jpainsymman.2009.06.001.

62. Allen RS, Thorn BE, Fisher SE, Gerstle J, Quarles K, Bourgeois MS, et al. Prescription and dosage of analgesic medication in relation to resident behaviors in the nursing home. J Am Geriatr Soc. 2003;51(4):534-8.

63. Alqualo-Costa R, Thomé GR, Perracini MR, Liebano RE. Low-level laser therapy and interferential current in patients with knee osteoarthritis: a randomized controlled trial protocol. Pain Manag. 2018;8(3):157-66.

64. Kamper SJ, Ostelo RWJG, Knol DL, Maher CG, de Vet HCW, Hancock MJ. Global Perceived Effect scales provided reliable assessments of health transition in people with musculoskeletal disorders, but ratings are strongly influenced by current status. J Clin Epidemiol [Internet]. 2010;63(7):760766.e1. Available from: http://dx.doi.org/10.1016/j.jclinepi.2009.09.009.

65. Kovacs FM, Abraira V, Royuela A, Corcoll J, Alegre L, Tomás M, et al. Minimum detectable and minimal clinically important changes for pain in patients with nonspecific neck pain. BMC Musculoskelet Disord. 2008;9(Mdc):1-9.

66. Rocha SP, De Benedetto MAC, Fernandez FHB, Gallian DMC. A trajetória da introdução e regulamentação da acupuntura no Brasil: Memórias de desafios e lutas. Cienc e Saude Coletiva. 2015;20(1):155-64.

\section{Tables}


Table 1

- Laser acupuncture parameters

\begin{tabular}{|c|c|}
\hline Parameters & Infrared laser \\
\hline Center wavelength [nm] & 808 \\
\hline Operating mode & Continuous wave \\
\hline Output Power [mW] & 80 \\
\hline Power density $\left[\mathrm{W} / \mathrm{cm}^{2}\right]$ & 1.14 \\
\hline Beam profile & unique \\
\hline Irradiance at target $\left[\mathrm{W} / \mathrm{cm}^{2}\right]$ & 1.14 \\
\hline Energy density $\left[\mathrm{J} / \mathrm{cm}^{2}\right]$ & 137.1 \\
\hline Total time per point [s] & 120 \\
\hline Radiant energy per point [J] & 9.6 \\
\hline Number of points irradiated & 8 \\
\hline Area irradiated $\left[\mathrm{cm}^{2}\right]$ & 0.07 \\
\hline Total radiant energy [J] & 76.8 \\
\hline Angle of application & $90^{\circ}$ \\
\hline Application technique & Contact \\
\hline
\end{tabular}

Figures 


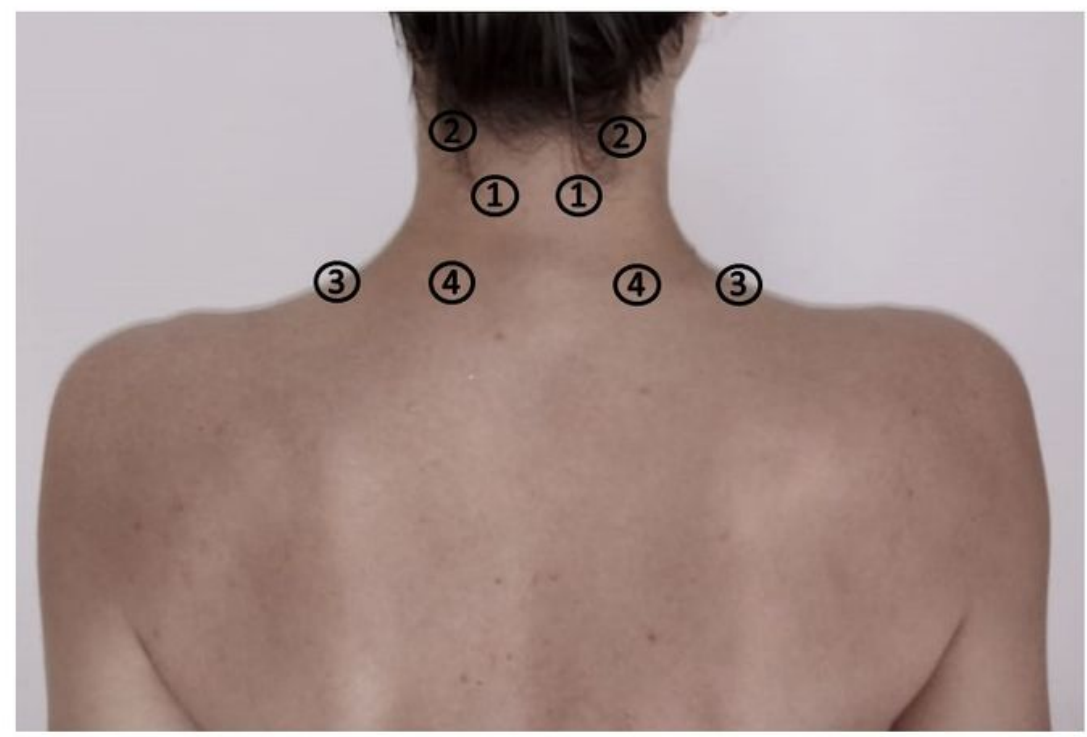

1.Tianzhu; 2.Fengchi; 3.Jianjing; 4. Jianzhongshu

\section{Figure 1}

Acupuncture points that will be used in therapies.

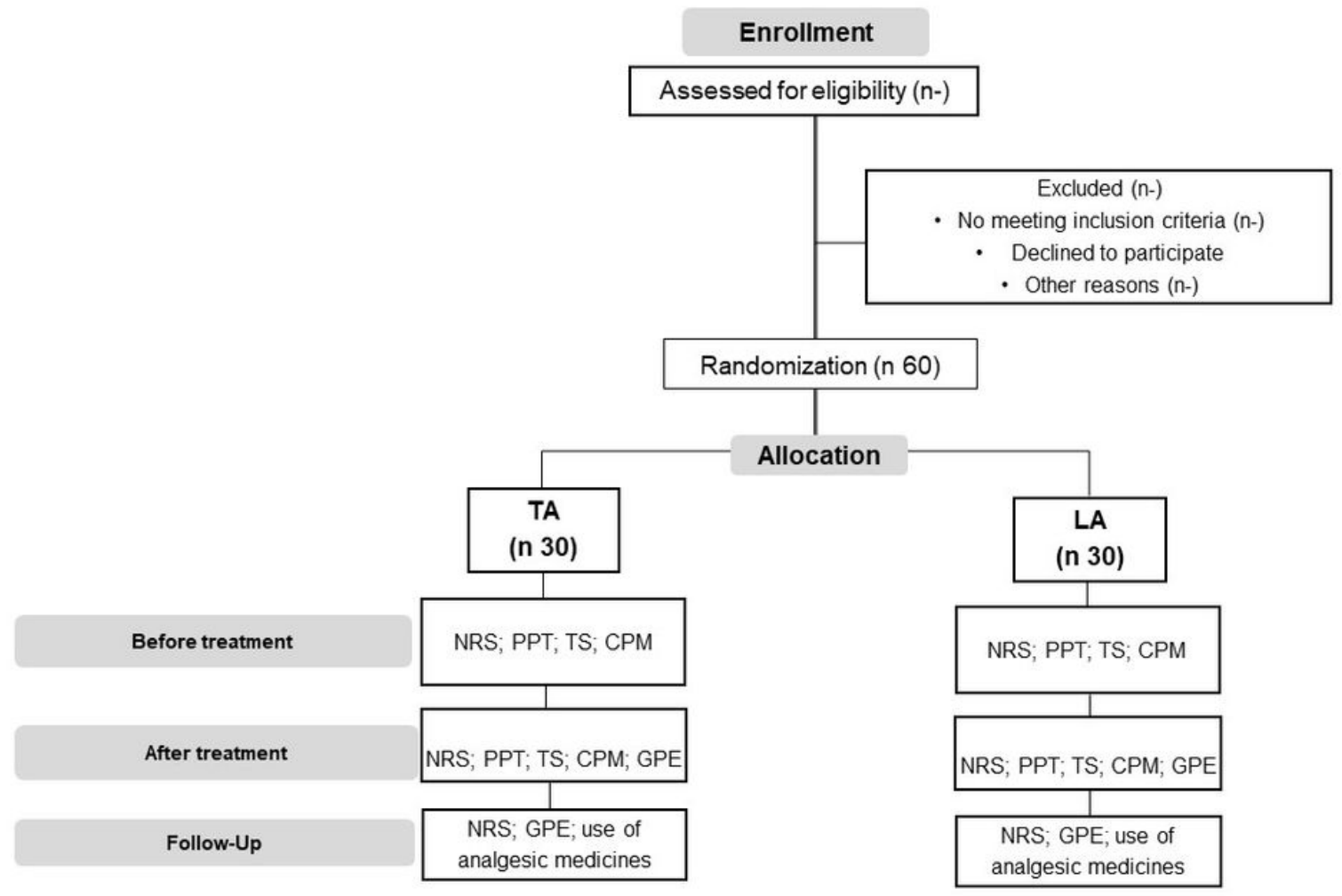


Figure 2

Flow diagram of the randomized clinical trial. NRS Numeric rating scale PPT Pressure pain threshold TS Temporal summation of pain CPM Conditioned pain modulation GPE Global perceived effect scale 